\title{
The Long Memory of the Jump Intensity of the Price Process
}

\author{
Yizhuang Tian', Dongyang Shi², Handong $\mathrm{Li}^{1 *}$ (1) \\ ${ }^{1}$ School of Systems Science, Beijing Normal University, Beijing, China \\ ${ }^{2}$ School of Government, Beijing Normal University, Beijing, China \\ Email: ${ }^{\star}$ lhd@bnu.edu.cn
}

How to cite this paper: Tian, Y.Z., Shi, D.Y. and Li, H.D. (2021) The Long Memory of the Jump Intensity of the Price Process. Journal of Mathematical Finance, 11, 176-189. https://doi.org/10.4236/jmf.2021.112009

Received: February 1, 2021

Accepted: March 29, 2021

Published: April 1, 2021

Copyright (c) 2021 by author(s) and Scientific Research Publishing Inc. This work is licensed under the Creative Commons Attribution International License (CC BY 4.0).

http://creativecommons.org/licenses/by/4.0/

\begin{abstract}
The impact of successive jumps in price process on volatility is very important. We study the nature of self-motivation in price process using data from China's stock market. Our empirical results suggest that: 1) Price jumps in China's stock market are generally self-motivated, i.e., price jumps are clustering. 2) The jump intensity of China's stock market is time-varying, and follows log-normal distribution, which indicates that the jump intensity is asymmetrical. 3) The jump intensities' sequence exhibits typical long memory.
\end{abstract}

\section{Keywords}

Price Jump, Self-Motivated, Jump Intensity, Hurst Index, Long Memory

\section{Introduction}

With the availability of high-frequency data, the study of dynamic behavior of volatility based on high-frequency data has become a focus of econophysics. A large number of studies have shown that volatility has remarkable clustering and long memory [1] [2] [3] [4]. The clustering and long memory of volatility are generally considered to be related to the jumps in the price process. Early researches into price jumps came from former works [5] [6]. In the last decade, the research focus of price jump has shifted from jump test to jump correlation [7]-[14]. Later research studied the correlation between jumps which they called self-motivation in a continuous semimartingale with jump component [15]. Another research team proposed methods to test jump self-motivation [16] [17]. In [15], the self-motivation of price jump is determined by a time-varying intensity process which can be expressed as a differential equation of time and counting process. Clearly, the dynamic behavior of intensity process dominates the degree 
of self-motivation of price jump. However, as far as we know, there is little research on the dynamic nature of the intensity process.

In this paper, our contribution is empirical. We use the jump self-motivation test [16] to study the phenomenon of price jump in China's stock market, and focus on the long memory of the intensity process of price jump. We find that the intensity of price jumps is generally long-memory in China's stock market. This not only explains the phenomenon of self-motivation between price jumps, but also deepens our understanding of the phenomenon of violent volatility in China's stock market.

The remainder of the paper is organized as follows. Section 2 formally introduces the jump detection method and self-motivation detection method. Section 3 presents the empirical analysis. Section 4 concludes. Appendix details the kernel database of statistics generated in our framework.

\section{Theory and Method}

\subsection{Price Jump Theory}

One-dimensional asset price process defined on probability space $\left(\Omega, \mathcal{F}_{t}, \mathcal{P}\right)$ could be presented as

$$
\log S(t)=\log S(0)+\int_{0}^{t} \mu_{s} \mathrm{~d} s+\int_{0}^{t} \sigma_{s} \mathrm{~d} W_{s}
$$

where $S(t)$ is asset price at time $t, \mu(t)$ for drift process, $\sigma(t)$ for realized market volatility, and $W(t)$ is standard Brownian process. The jump component is introduced to obtain the following formula:

$$
\begin{aligned}
\log S(t)= & \log S(0)+\int_{0}^{t} \mu_{s} \mathrm{~d} s+\int_{0}^{t} \sigma_{s} \mathrm{~d} W_{s}+h(\delta) * s *\left(b^{s}-v^{s}\right)_{t} \\
& +(\delta-h(\delta)) * b_{t}^{s}
\end{aligned}
$$

where $h$ is truncation function, $b^{s}$ and $v^{s}$ are poisson distribution estimate in $\mathbb{R} \times E, \delta$ is predictable function. For Equation (2), assumptions follow:

Assumption 1: $b$ and $\sigma$ are locally bounded.

Assumption 2: $v^{s}(\omega, \mathrm{d} t, \mathrm{~d} s)=\mathrm{d} t \otimes F_{s}(\omega, \mathrm{d} s)$, where $F_{s}$ is a predictable random measure. Set three non-random numbers $\left(\beta \in(0,2), \beta^{\prime} \in[0, \beta)\right.$ and $\gamma>0)$ and a locally bounded process $\left(L_{t} \geq 1\right)$ to have $F_{t}=F_{t}^{\prime}+F_{t}^{\prime \prime}$ for all $(\omega, \mathrm{d} t)$, where

$$
F_{t}^{\prime}(\omega, \mathrm{d} x)=f_{t}^{\prime}(x) \lambda_{t}-(\omega) \mathrm{d} x, 0<\lambda_{t}(\omega) \leq L_{t},
$$

1)

$$
f_{t}^{\prime}(x)=\frac{1+|x|^{\gamma} h_{t}(x)}{|x|^{1+\beta}}, 1+|x|^{\gamma} h_{t}(x) \geq 0,\left|h_{t}(x)\right| \leq L_{t} ;
$$

2) $F_{t}^{\prime \prime}$ is a singular measure and $F_{t}^{\prime}$ satisfies $\int\left(|x|^{\beta^{\prime}} \wedge 1\right) F_{t}^{\prime \prime}(\mathrm{d} x) \leq L_{t}$;

3) $\lambda_{t}$ is a positive Itô semimartingale process,

$\lambda_{t}=\lambda_{0}+\int_{0}^{t} \mu_{s}^{\prime} \mathrm{d} s+\int_{0}^{t} \sigma_{s}^{\prime} \mathrm{d} W_{s}+\int_{0}^{t} \sigma_{s}^{\prime \prime} \mathrm{d} \tilde{B}_{s}+\delta^{\prime} \times \mu_{t}^{x}+\delta^{\prime \prime} \times \mu_{t}^{\perp x}$, where $\tilde{B}$ is a standard Brownian process independent from $W, \mu_{t}^{x}$ is orthogonal to $\mu_{t}^{\perp x}$, and $\delta^{\prime}, \delta^{\prime \prime}$ are predictable.

On the basis of asset price process above, define asset return at time $t_{i}$ as 


$$
r_{t_{i}}:
$$

When applying to discrete data, $T$ refers to total number of minutes $(T \in \mathbb{N})$. Let sampling frequency be $\Delta_{n}=T / n$, where sample size is $n$ and $t=i *$ $\Delta_{n}(i \in \mathbb{N})$. Let $X_{i}=\log \left(S_{i * \Delta_{n}}\right)$, asset return can be defined as:

$$
r_{\Delta_{n}, i}=\log \left(S_{i * \Delta_{n}}\right)-\log \left(S_{(i-1) * \Delta_{n}}\right) \triangleq \Delta_{i}^{n} X
$$

\subsection{Price Jump Self-Motivation Test}

In this paper, we use the self-motivation test method of price jump to make empirical analysis [16]. Boswijk extends the activity of price jump to infinite jump. The self-motivation is defined as

$$
\sum_{0 \leq t \leq T} \Delta \lambda_{t} 1_{\left\{\Delta \lambda_{t} \geq 0,\left|\Delta X_{t}\right| \neq 0\right\}}>0
$$

where $T$ stands for a total length of the sample, $X_{t}=\log \left(S_{t}\right)$, and $\lambda_{t}$ is density of jump arrivals at $t$.

Equation (5) shows, within the data range, the jump arrival density also changes in a non-negative way when the price changes, which means there's self-motivation in the jumps.

Choose a proper window size $k_{n}$, the jump intensity $\hat{\lambda}\left(k_{n}\right)_{i}$ in $i$ th window size

$$
\hat{\lambda}\left(k_{n}\right)_{i}=\frac{\Delta_{n}^{\varpi \hat{\beta}}}{k_{n} \Delta_{n}} \sum_{j=i+1}^{i+k_{n}} g\left(\frac{\left|r_{\Delta_{n}, j}\right|}{\alpha \Delta_{n}^{\sigma}}\right) \frac{\alpha^{\hat{\beta}}}{C_{\hat{\beta}}(1)}
$$

In Equation (6), $0<\varpi<\frac{1}{2}, \alpha$ is the standard deviation of the income distribution and function $g(\cdot)$ has following 3 forms:

$$
\begin{gathered}
g_{0}(x)=1_{\{x>1\}} \\
g_{1}(x)=\left\{\begin{array}{l}
|x|^{p},|x| \leq 1 \\
1,|x|>1
\end{array}\right. \\
g_{2}(x)=\left\{\begin{array}{l}
c^{-1}|x|^{p},|x| \leq a \\
c^{-1}\left(a^{p}+\frac{p a^{p-1}}{2(b-a)}\left((b-a)^{2}-(|x|-b)^{2}\right)\right), a \leq|x| \leq b \\
1,|x| \geq b
\end{array}\right.
\end{gathered}
$$

In Equation (9), $c=a^{p}+\frac{p a^{p-1}(b-a)}{2}$, and when $a=b=1, g_{2}(x)=g_{1}(x)$. In applications, $g(\cdot)$ can be taken as any of the three forms.

$C_{\hat{\beta}}(k)$ is defined as

$$
C_{\beta}(k)=\int_{0}^{\infty}(g(x))^{k} / x^{1+\beta} \mathrm{d} x
$$


where the estimation of $\beta$ is

$$
\hat{\beta}_{n}\left(t, \varpi, \alpha, \alpha^{\prime}\right)=\log \frac{V(\varpi, \alpha, g)_{t}^{n}}{V\left(\varpi, \alpha^{\prime}, g\right)_{t}^{n}} / \log \frac{\alpha^{\prime}}{\alpha}
$$

where $\alpha^{\prime}=2 * \alpha$, and $V(\varpi, \alpha, g)$ :

$$
V(\varpi, \alpha, g)_{t}^{n}=\sum_{i=1}^{n} g\left(\frac{r_{\Delta_{n} i}}{\alpha \Delta_{n}^{\sigma}}\right)
$$

According to Equation (5), statistics $U\left(H, k_{n}\right)_{T}$ and $U\left(G, k_{n}\right)_{T}$ :

$$
\begin{aligned}
U\left(H, k_{n}\right)_{T}= & \sum_{i=k_{n}+1}^{[T / \Delta n]-k_{n}} H\left(X_{(i-1) \Delta_{n}}, X_{i \Delta_{n}}, \hat{\lambda}\left(k_{n}\right)_{i-k_{n}-1}, \hat{\lambda}\left(k_{n}\right)_{i}\right) \\
& \times 1_{\left\{||_{\Delta_{n}, i} \mid>\alpha \Delta_{n}^{\sigma}\right\}} \times 1_{\left\{\left|r_{\Delta_{n}, i}\right| \in \mathcal{E}\{\{0\}\}\right.}
\end{aligned}
$$

where $\epsilon=\bar{r} \sqrt{\theta} \Delta_{n}^{\sigma}$. The $H$ function is expressed as following:

$$
H\left(x_{1}, x_{2}, y_{1}, y_{2}\right)=\left|x_{2}-x_{1}\right|^{p} h_{2}\left(y_{1}, y_{2}\right)
$$

In Equation (14), $p$ is usually 2 or 4 . And $h_{2}\left(y_{1}, y_{2}\right)$ could be expressed as:

$$
h_{2}\left(y_{1}, y_{2}\right)=\left\{\begin{array}{l}
\exp \left(-1 /\left(y_{2}-y_{1}\right)\right) \text { if } y_{2}>y_{1} \\
0 \text { if } y_{2} \leq y_{1}
\end{array}\right.
$$

Statistic $U\left(G, k_{n}\right)_{T}$ is showed in the following form

$$
\begin{aligned}
U\left(G, k_{n}\right)_{T}= & \sum_{i=k_{n}+1}^{[T / \Delta n]-k_{n}} G\left(X_{(i-1) \Delta_{n}}, X_{i \Delta_{n}}, \hat{\lambda}\left(k_{n}\right)_{i-k_{n}-1}, \hat{\lambda}\left(k_{n}\right)_{i}\right) \\
& \times 1_{\left\{||_{\Delta_{n}, i} \mid>\alpha \Delta_{n}^{\Phi}\right\}} \times 1_{\left\{||_{\Delta_{n}, i} \mid \in \mathcal{E} \backslash\{0\}\right\}}
\end{aligned}
$$

where $G$ function is

$$
G\left(x_{1}, x_{2}, y_{1}, y_{2}\right)=\frac{\alpha^{\beta} C_{\beta}(2)}{\left(C_{\beta}(1)\right)^{2}}\left(y_{1} H_{3}^{\prime}\left(x_{1}, x_{2}, y_{1}, y_{2}\right)^{2}+y_{2} H_{4}^{\prime}\left(x_{1}, x_{2}, y_{1}, y_{2}\right)^{2}\right)
$$

where $H_{3}^{\prime}\left(x_{1}, x_{2}, y_{1}, y_{2}\right)$ and $H_{4}^{\prime}\left(x_{1}, x_{2}, y_{1}, y_{2}\right)$ are the first partial derivatives of function $H\left(x_{1}, x_{2}, y_{1}, y_{2}\right)$ with respect to $y_{1}, y_{2}$. Statistics $U\left(H, k_{n}\right)_{T}$ and $U\left(G, k_{n}\right)_{T}$ are continuous parameters with finite variance, and have the following asymptotic properties:

$$
t_{n}:=\sqrt{\frac{k_{n} \Delta_{n}}{\Delta_{n}^{\sigma \beta}}} \frac{U\left(H, k_{n}\right)_{T}}{\sqrt{U\left(G, k_{n}\right)_{T}}}\left\{\begin{array}{l}
\stackrel{\mathbb{P}}{\longrightarrow}-\infty \quad \omega \in \Omega_{T}^{-} \\
\stackrel{L_{s t}}{\longrightarrow} \mathcal{N}(0,1) \quad \omega \in \Omega_{T}^{0} \\
\stackrel{\mathbb{P}}{\longrightarrow}+\infty \quad \omega \in \Omega_{T}^{\div}
\end{array}\right.
$$

Under the null hypothesis that price is not self-motivated, we could apply the above statistics to do hypothesis testing. However, under the null hypothesis that price is self-motivated, $U\left(H, k_{n}\right)$ could not be estimated as a finite sample error. Therefore, we need to reconstruct relative error statistic as following:

$$
R_{n}=\frac{U\left(H, \omega k_{n}\right)_{T}-U\left(H, k_{n}\right)_{T}}{U\left(H, k_{n}\right)_{T}}
$$

Relative error $R_{n}$ has following asymptotic properties: 


$$
\left\{\begin{array}{l}
R_{n} \stackrel{\mathbb{P}}{\longrightarrow} 0 \quad \omega \in \Omega_{T}^{\text {self }} \\
R_{n} \stackrel{L_{\text {st }}}{\longrightarrow} \frac{\overline{\mathcal{U}}_{T}^{\prime}}{\overline{\mathcal{U}}_{T}}-1 \neq 0 \quad \omega \in \Omega_{T}^{0}
\end{array}\right.
$$

where $L_{\text {st }}$ denotes stable convergence in law.

And its rejection region is

$$
C_{n}=\left\{\sqrt{\frac{k_{n} \Delta_{n}}{\Delta_{n}^{\sigma \beta}}} \frac{U\left(H, k_{n}\right)_{T}}{\sqrt{U\left(G, k_{n}\right)_{T}}}>Z_{\tilde{a}}\right\}
$$

where $Z_{a}$ is quantile of the standard normal distribution, and statistic $V_{n}$ is shown as following

$$
V_{n}=\frac{\Delta_{n}^{\pi \beta}}{k_{n} \Delta_{n}} \frac{(\omega-1) U\left(G, k_{n}\right)_{T}}{\omega\left(U\left(H, k_{n}\right)_{T}\right)^{2}}
$$

\section{Empirical Study}

\subsection{Data Source}

In empirical analysis, we selected the CSI300 index and its constituent stocks of China stock market from January to December 2015. Twenty-three stocks were randomly sampled from CSI300 index (SH000300). The names and codes of 23 stocks were shown in Table 1, and the 1-minute price data and CSI300 index (SH000300) were obtained.

Resample 1-minute price into 5-minute price and smooth the return series:

$$
\begin{gathered}
X_{i}=\log S_{5_{* i}}, \quad i \in(0, n) \\
r_{i}=\frac{1}{5} \sum_{k=1}^{5}\left(\log S_{5+k}-\log S_{k}\right)
\end{gathered}
$$

Since we connect all the 1-minute price series, the overnight effect and weekend effect should be taken into consideration, which could have a negative impact on our result of detected jumps. We applied such method to get rid of the unexpected effects:

$$
r_{G t}=\frac{r_{g t}-\mu_{g t}}{\sigma_{g t}} \sigma+\mu
$$

Table 1. Information of 30 stocks selected.

\begin{tabular}{cccccc}
\hline Name & Code & Name & Code & Name & Code \\
\hline CSI300 & SH000300 & CGGC & SH600068 & PETROCHINA & SH601857 \\
HD MEDICINE & SH000963 & NARI-TECH & SH600406 & JDCMOLY & SH601958 \\
HPI & SH600011 & KWEICHOW & SH600519 & PAB & SZ000001 \\
SIPG & SH600018 & XTC & SH600549 & TCL & SZ000100 \\
BAO STEEL & SH600019 & COOEC & SH600583 & WEICHAI POWER & SZ000338 \\
SEP & SH600021 & FYG & SH600660 & FINANCIAL STREET & SZ000402 \\
PRE & SH600048 & DAQIN RAILWAY & SH601006 & YUNNAN BAIYAO & SZ000538 \\
CHINA & SH600050 & BANKCOMM & SH601328 & SHUANGHUI & SZ000895 \\
UNICOM & SHNANG & & & &
\end{tabular}


where $r_{g t}, \mu_{g t}, \sigma_{g t}$ represent overnight return, average overnight return and standard deviation of overnight return before operation. $r_{G t}$ is the overnight return after operation, $\sigma$ is standard deviation of all non-overnight returns and $\mu$ is the average of non-overnight return. The weekend effect is removed in the same way.

After getting rid of the effects, we obtain the price and return series, two samples (CSI300 index, SH60048) is shown as follows.

Figure 1 shows the index and randomly selected stock had experienced sharp price fluctuations in the second half of 2015, and return distribution is found to be more of leptokurtosis. The statistics of returns is listed in Table 2.

Table 2 shows that the index yield and individual stock yields both have a peek skewed to the left. And individual stock return's standard deviation is significantly greater than the index yield.

\subsection{Price Jump Self-Motivation Test}

In this section, we test the self-motivation of price jumps of CSI300 index and constituent stocks on 5 -min price series. Set $k_{n}=10, \varpi=\frac{1}{5}, p=4, \omega=2$,
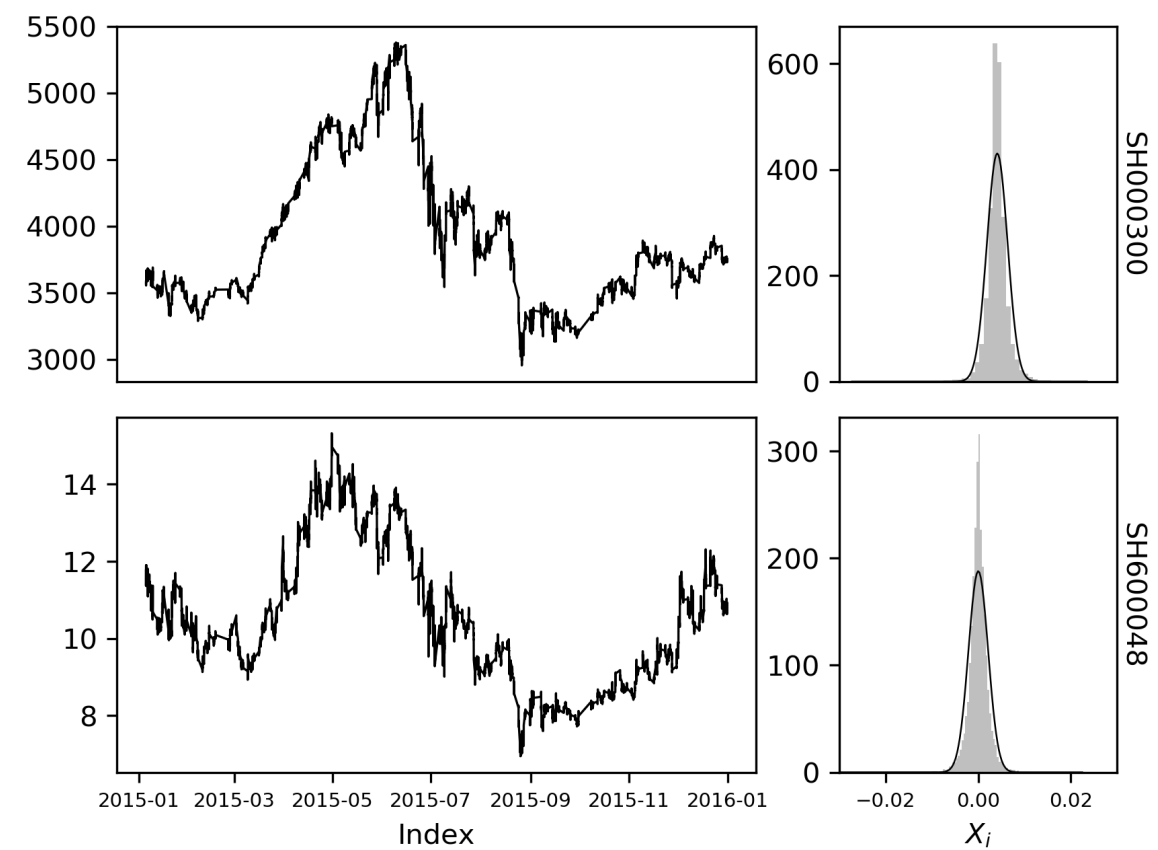

Figure 1. Price series and return distributions of SH00300 and SH60048 in year 2015.

Table 2. Statistics of returns of CSI300 index and SH60048.

\begin{tabular}{ccc}
\hline & SH000300 & SH000963 \\
\hline Mean & 0.0000286 & 0.0000368 \\
Std. & 0.0000743 & 0.002618 \\
Skew. & -0.034909 & -0.271355 \\
Kurt. & 11.35313 & 13.91738 \\
\hline
\end{tabular}


$\theta=\frac{1}{16}$. Function $g(\cdot)$ is set in the simple form of Equation (8). CSI300 index and 23 constituent stocks all passed the test under the null hypothesis that price jump is self-motivated. The p-value for CSI300 index is 0.3879 and p-values of 23 constituent stocks are showed in Table 3 . The result implies the price jump in China stock market has significant self-motivation.

The estimates of jump arrival intensities $\hat{\lambda}$ 's of CSI300 index and constituent stocks are critical parameters in self-motivation test. The $\hat{\lambda}$ 's of CSI300 index and 3 stocks (SH000963, SH600011 and SH600018) in 2015 is shown as follows (the $\hat{\lambda}$ 's of other 20 stocks' are displayed in Appendix 1).

In Figure 2, each $\hat{\lambda}$ value is a dot. Considering the date time index is too

Table 3. Hurst index of CSI300 index, constituent stocks and MEAN.

\begin{tabular}{cccc}
\hline Code & Hurst Index & Code & Hurst Index \\
\hline SH000300 & 0.8710882 & SH000963 & 0.8849673 \\
SH600011 & 0.9014997 & SH600018 & 0.8531737 \\
SH600019 & 0.8835869 & SH600021 & 0.8617589 \\
SH600048 & 0.7457267 & SH600050 & 0.8404174 \\
SH600068 & 0.8452361 & SH600406 & 0.8815753 \\
SH600519 & 0.877194 & SH600549 & 0.8455916 \\
SH600583 & 0.8936722 & SH600660 & 0.8064734 \\
SH601006 & 0.8731213 & SH601328 & 0.8386584 \\
SH601857 & 0.8339011 & SH601958 & 0.852093 \\
SZ000001 & 0.7710014 & SZ000100 & 0.8499707 \\
SZ000338 & 0.8469565 & SZ000402 & 0.8098829 \\
SZ000538 & 0.858791 & SZ000895 & 0.8585242 \\
MEAN & 0.8892194 & & \\
\hline
\end{tabular}
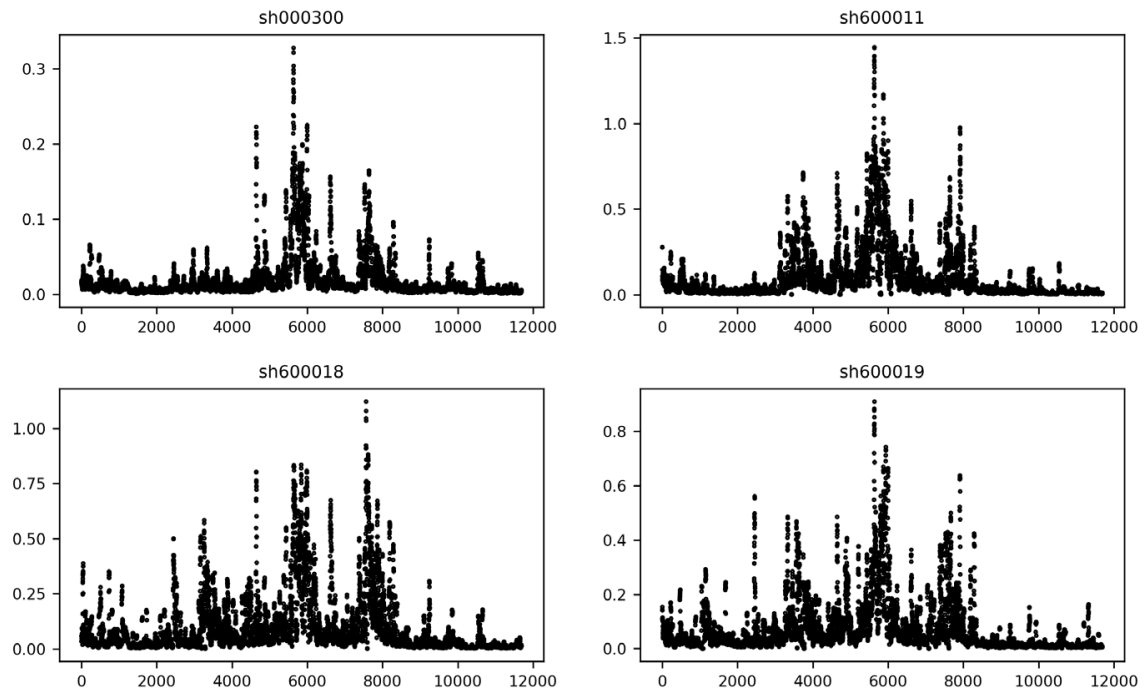

Figure 2. $\hat{\lambda}$ 's of CSI300 index and 3 stocks (SH600011, SH600018, and SH600019) in year 2015 . 
long (i.e. 09:35, 2015/01/05), transform the datetime into serial number ranging from 1 to 12,000 . Since the sampling frequency is 5 minutes, each number on the abscissa represents a 5 -minute return. From Equation (6), $\hat{\lambda}\left(k_{n}\right)_{i}$ is calculated using $k_{n}$ returns in $i$ th window. Combing Equation (7), ith window and $(i+1)$ th window have only one different return value, so it's probable that $\hat{\lambda}\left(k_{n}\right)_{i} \approx \hat{\lambda}\left(k_{n}\right)_{i+1}$. For this reason, it's reasonable to have continuously equal $\hat{\lambda}$ in Figure 2 .

In Figure 2, each subfigure has a peak at serial number 6000, which is equal to early June in 2015. At that time, there was a terrible crash in China's Stock Market with sharp fluctuations.

\subsection{Discussion}

Each $\hat{\lambda}$ is an estimate of jump arrival intensity and it's time-varying. To study the nature of $\hat{\lambda}$ 's, we analyzed the distribution characteristics of jump arrival intensity of 23 constituent stocks. Frequency histograms of the $\hat{\lambda}$ 's of CSI300 index and 3 individual stocks (SH600011, SH600018, and SH600019) in 2015 are displayed in Figure 3. The other 20 stocks are detailed in Appendix 2.

In Figure 3 , abscissa represents the value of $\hat{\lambda}$ 's and ordinate is the frequency of $\hat{\lambda}$ 's. It's shown that the jump intensities of CSI300 index and 3 individual stocks have similar density distribution, reflecting that the inherent similarity in the intensity of price jumps. By averaging the jump intensity of these 24 index and stocks, the robust and common distribution characteristics of jump intensity can be obtained. The average jump arrival intensity of 30 stocks and its distribution figure are shown below.

In Figure 4, left subfigure is average $\hat{\lambda}$ 's of 24 index and stocks. Similar to Figure 2, each $\hat{\lambda}$ value is a dot, abscissa value is serial number of $\hat{\lambda}$ sequence and ordinate value is the frequency of $\hat{\lambda}$ 's. There's also a peek at serial number
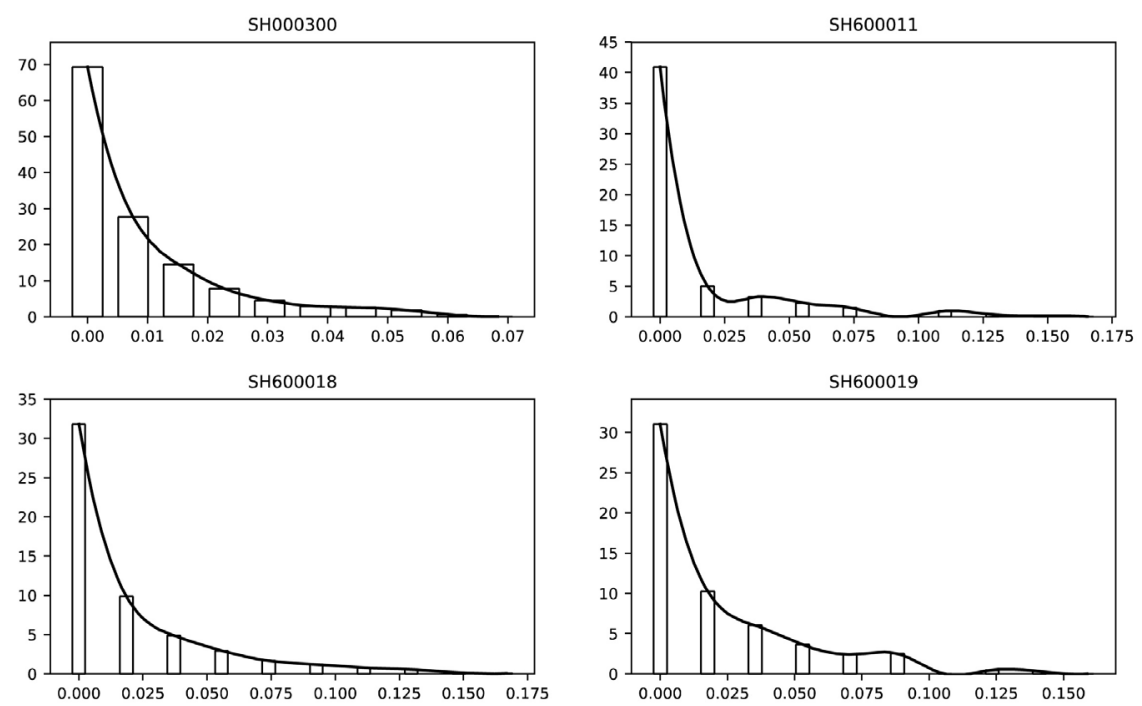

Figure 3. Frequency histograms of $\hat{\lambda}$ 's of CSI300 index and 3 individual stocks (SH600011, SH600018, and SH600019). 

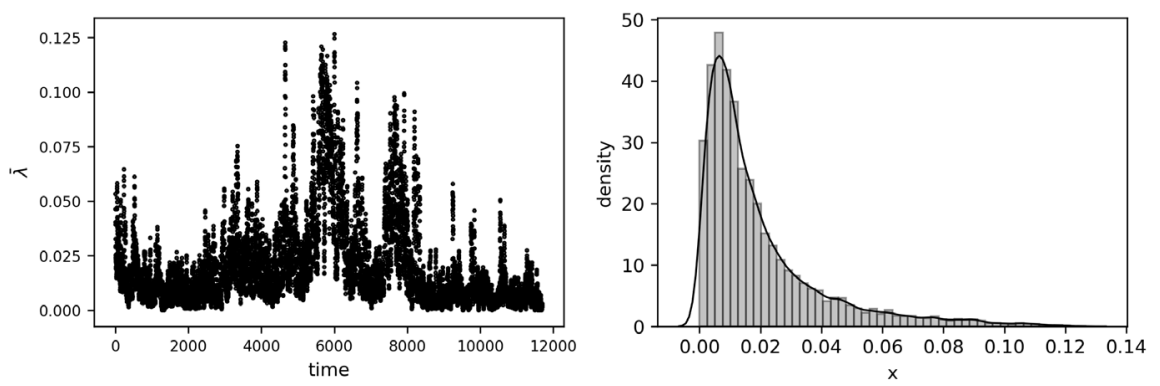

Figure 4. Average jump arrival intensity of 30 stocks and its distribution.

6000 , showing that China's stock market has the highest jumping intensity in the average sense during this period. It corresponded with the fact that a crash happened in the middle of 2015 in China's stock market.

Right subfigure in Figure 4 is the density distribution of averaging $\hat{\lambda}$ 's. It tends to be log-normal. So we use KS test to identify it. The result shows that the jump intensities pass log-normal hypothesis and fitting results are displayed in Figure 5.

Figure 5 shows the difference between the fitted empirical distribution and the lognormal distribution. The results indicate that the distribution of averaging $\hat{\lambda}$ 's is very similar to that of lognormal distribution. Log-normal distribution indicates that the distribution of time-varying arrival intensities is asymmetric, which is reflected in the market that the higher the frequency of price jump, the wider the range of frequency change. This suggests that there may be a long memory of the jump intensities. Therefore, we will use the detrended fluctuation analysis (DFA) method to study the long memory of intensities' series. The DFA method proposed by Peng (1994) is a nonparametric estimation method of generalized Hurst index and it can be used to detect the long-range correlation of non-stationary time series.

We calculated the Hurst indices of $\hat{\lambda}$ 's by using DFA. This method can calculate the generalized Hurst index by removing the trend term. If the Hurst exponent is between 0.5 and 1 , we can conclude that the sequence has long memory, and the closer it is to 1 , the stronger the long memory. The results are shown in Table 3.

From Table 3, Hurst indices of $\hat{\lambda}$ series are greater than 0.5 , and most of them are greater than 0.8 , very close to 1 . This shows that the jump intensities' series of the high-frequency price series in China's stock market have very strong long memory. This not only indicates that there is a correlation between the price jumps in China's stock market, but also present the clustering effect of jumps, which is long memory.

\section{Conclusions}

\subsection{Empirical Conclusions}

As an emerging market, China's stock market shows more volatility. The jumping phenomenon in the high-frequency price process is one of the main causes 

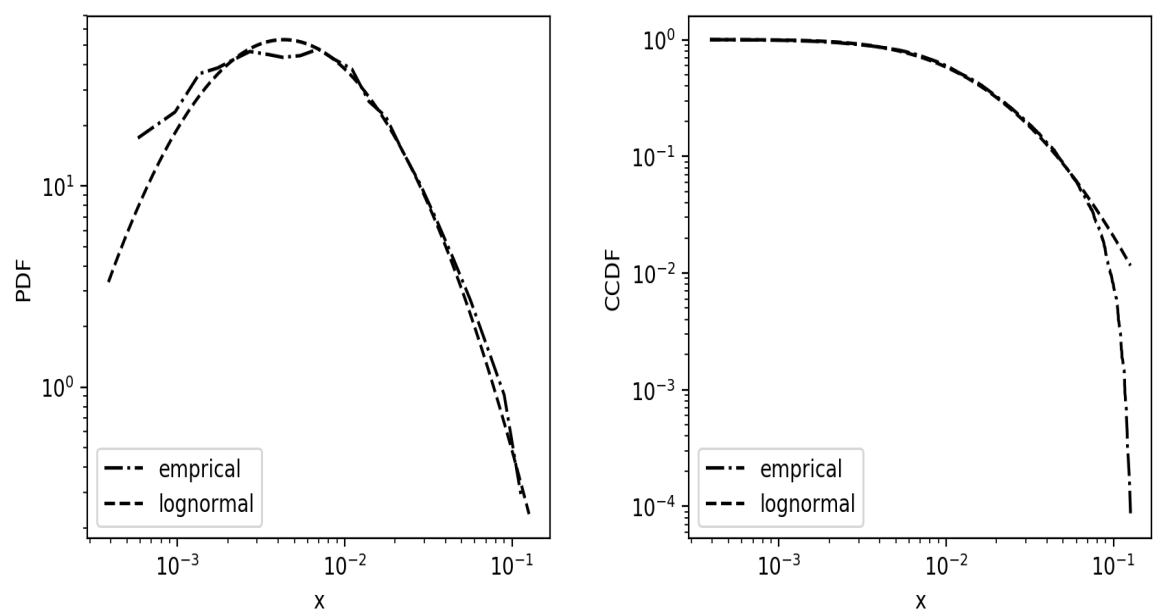

Figure 5. Log-normal fitting curve of $\hat{\lambda}$ 's.

of the market volatility. In this paper, we choose the CSI300 index and 23 stocks that are randomly selected in the constituents of CSI300 index as the representative of China's stock market, and take the 1-minute high-frequency price data of 2015, which is highly volatile in China's stock market, as samples, and then resamples into 5-minute return series to conduct empirical analysis such as jump test and jump self-motivation test. Our empirical results show that:

1) It's clear that the number of jumps of an individual stock is more than the one of an index in high-frequency dataset. This is consistent with many studies. When individual stocks jump, the index doesn't jump much, showing that the probability that the index resonates with individual stocks is low. When the index jumps, the proportion of individual stocks that jump is not high, either. Within portfolio theory, the jumps of the index could be regarded as a co-jump contributed by individual stocks' heterogeneous jumps after fully smoothing, which means the connections between stocks and the index could be implied in called co-jumps. However, co-jumps would also be covered by heterogenous jumps and random noises of individual stocks.

2) In the empirical analysis of high-frequency price series of the CSI300 index and selected 23 constituent stocks, the index and the stocks all passed the test under the null hypothesis that there is a self-motivation jump in the price process, indicating that the price jump in China's stock market is generally self-motivated. And the fluctuations of jump intensity estimates used in the self-motivation test basically correspond to current situation in last decade.

3) In the analysis of time-varying jump intensity series, we found that the jump intensity series of asset price in China's stock market is approximately lognormal distribution, which indicates that the change of jump intensity is asymmetric. At the same time, we also found that the price jump intensity series shows strong long-term memory, which indicates that the self-motivation phenomenon of price jump intensity is long-term related. This can provide a new perspective for us to understand the risk in China's stock market. 


\subsection{Future Expectations}

Our work has shown a sound result that self-motivated jumps exist in China's stock market, though we need more precise price stochastic process forms. There are still some facts needed focused in future:

1) How the log-normal distributed price processes interact and contribute to the index fluctuations. Reconsidering the price generation tools and theoretical derivations is advised.

2) China's stock market has been experiencing subtle and swift adjustments in many ways in recent years, including new market trade instruments and more specific regulatory policies. The uncertainty comes from the structure of market and would lead to more unexpected fluctuations or severe changes, though it's in belief that China's stock market would keep open and integrate more with international financial markets. Finding a straightforward toolbox in dealing with the more common and frequent jumps is necessary and what we're devoted to.

Based on the results of this paper, subsequent research will focus on the generating mechanism of the price jump process, so as to improve the price process and make the jump terms in the price process include the co-jumps and the jump self-motivation part. Then we could discuss the jumps and co-jumps in more detailed situations and provide a reasonable explanation to our interests.

\section{Acknowledgements}

This work is supported by the National Natural Science Foundation of China (Grant No. 71671017).

\section{Declaration of Interest Statement}

No potential conflict of interest was reported by the authors.

\section{References}

[1] Maasoumi, E. and McAleer, M. (2008) Realized Volatility and Long Memory: An Overview. Econometric Reviews, 27, 1-9. https://doi.org/10.1080/07474930701853459

[2] Kim, S. and Eom, C. (2008) Long-Term Memory and Volatility Clustering in High-Frequency Price Changes. Physica A: Statistical Mechanics and Its Applications, 387, 1247-1254. https://doi.org/10.1016/j.physa.2007.08.061

[3] Choi, K., Yu, W.-C. and Zivot, E. (2010) Long Memory Versus Structural Breaks in Modeling and Forecasting Realized Volatility. Journal of International Money and Finance, 29, 857-875. https://doi.org/10.1016/j.jimonfin.2009.12.001

[4] Degiannakis, S. and Floros, C. (2016) Intra-Day Realized Volatility for European and USA Stock Indices. Global Finance Journal, 29, 24-41. https://doi.org/10.1016/j.gfj.2015.05.002

[5] Merton, R.C. (1976) Option Pricing when Underlying Stock Returns Are Discontinuous. Journal of Financial Economics, 3, 125-144. https://doi.org/10.1016/0304-405X(76)90022-2

[6] Tankov, P. (2003) Financial Modelling with Jump Processes. CRC Press, New York, 552. https://doi.org/10.1201/9780203485217 
[7] Lee, S.S. and Mykland, P.A. (2008) Jumps in Financial Markets: A New Nonparametric Test and Jump Dynamics. The Review of Financial Studies, 21, 2535-2563. https://doi.org/10.1093/rfs/hhm056

[8] Aït-Sahalia, Y. and Jacod, J. (2009) Estimating the Degree of Activity of Jumps in High Frequency Data. The Annals of Statistics, 37, 2202-2244.

https://doi.org/10.1214/08-AOS640

[9] Aït-Sahalia, Y. and Jacod, J. (2009) Testing for Jumps in a Discretely Observed Process. The Annals of Statistics, 37, 184-222. https://doi.org/10.1214/07-AOS568

[10] Aït-Sahalia, Y. and Jacod, J. (2011) Testing Whether Jumps have Finite or Infinite Activity. The Annals of Statistics, 39, 1689-1719.

https://doi.org/10.1214/11-AOS873

[11] Ait-Sahalia, Y. and Jacod, J. (2012) Identifying the Successive Blumenthal-Getoor Indices of a Discretely Observed Process. The Annals of Statistics, 40, 1430-1464. https://doi.org/10.1214/12-AOS976

[12] Aït-Sahalia, Y., Jacod, J. and Li, J. (2012) Testing for Jumps in Noisy High Frequency Data. Journal of Econometrics, 168, 207-222. https://doi.org/10.1016/j.jeconom.2011.12.004

[13] Todorov, V. and Tauchen, G. (2010) Activity Signature Functions for High-Frequency Data Analysis. Journal of Econometrics, 154, 125-138.

https://doi.org/10.1016/j.jeconom.2009.06.009

[14] Jing, B.-Y., et al. (2012) On the Jump Activity Index for Semimartingales. Journal of Econometrics, 166, 213-223. https://doi.org/10.1016/j.jeconom.2011.09.036

[15] Aït-Sahalia, Y., Cacho-Diaz, J. and Laeven, R.J. (2015) Modeling Financial Contagion Using Mutually Exciting Jump Processes. Journal of Financial Economics, 117, 585-606. https://doi.org/10.1016/j.jfineco.2015.03.002

[16] Boswijk, H.P., Laeven, R.J. and Yang, X. (2018) Testing for Self-Excitation in Jumps. Journal of Econometrics, 203, 256-266.

https://doi.org/10.1016/j.jeconom.2017.11.007

[17] Corradi, V., Silvapulle, M.J. and Swanson, N.R. (2018) Testing for Jumps and Jump Intensity Path Dependence. Journal of Econometrics, 204, 248-267.

https://doi.org/10.1016/j.jeconom.2018.02.004 


\section{Appendix 1}
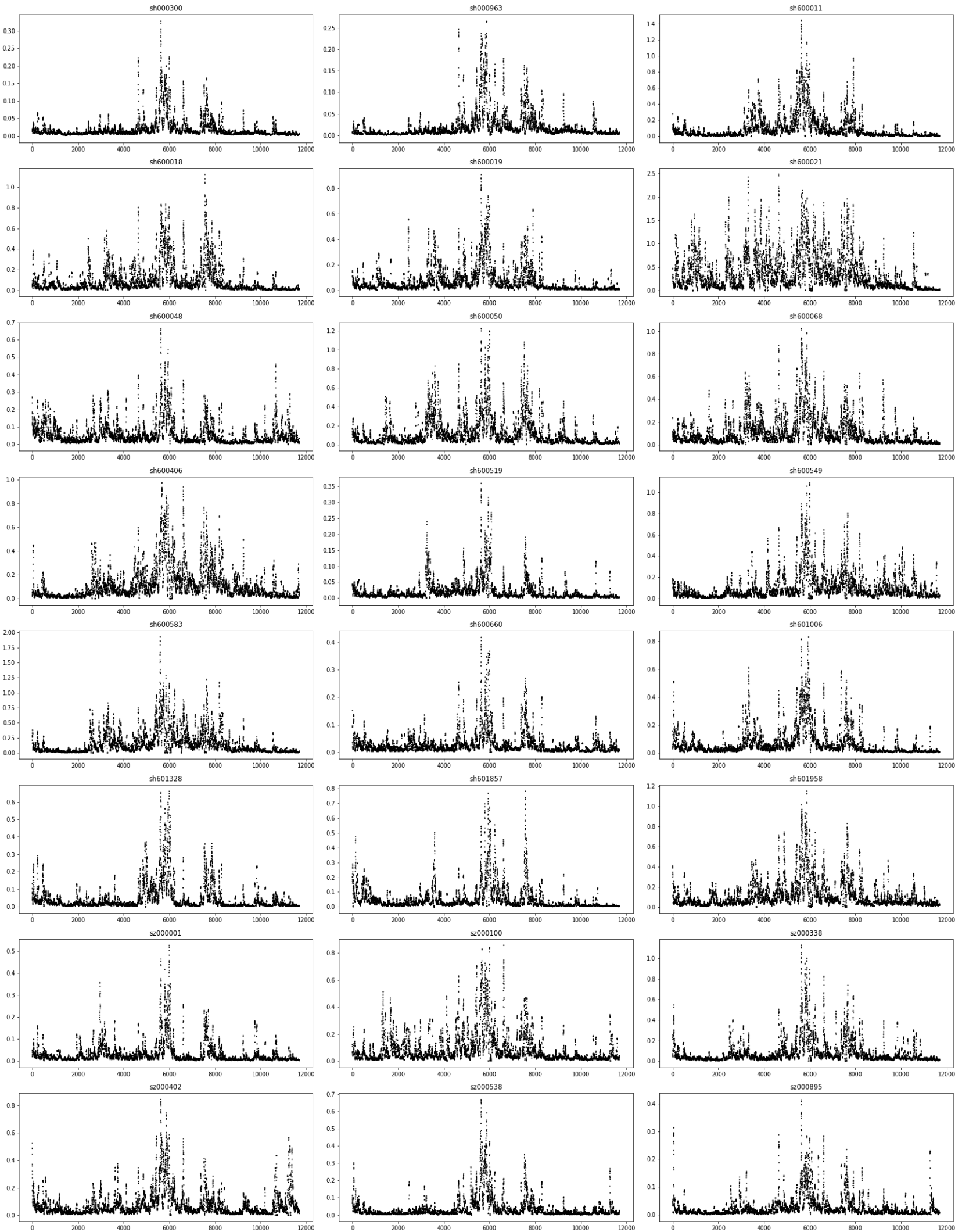

Figure A1. $\hat{\lambda}$ of the index and 3 constituent stocks. 


\section{Appendix 2}
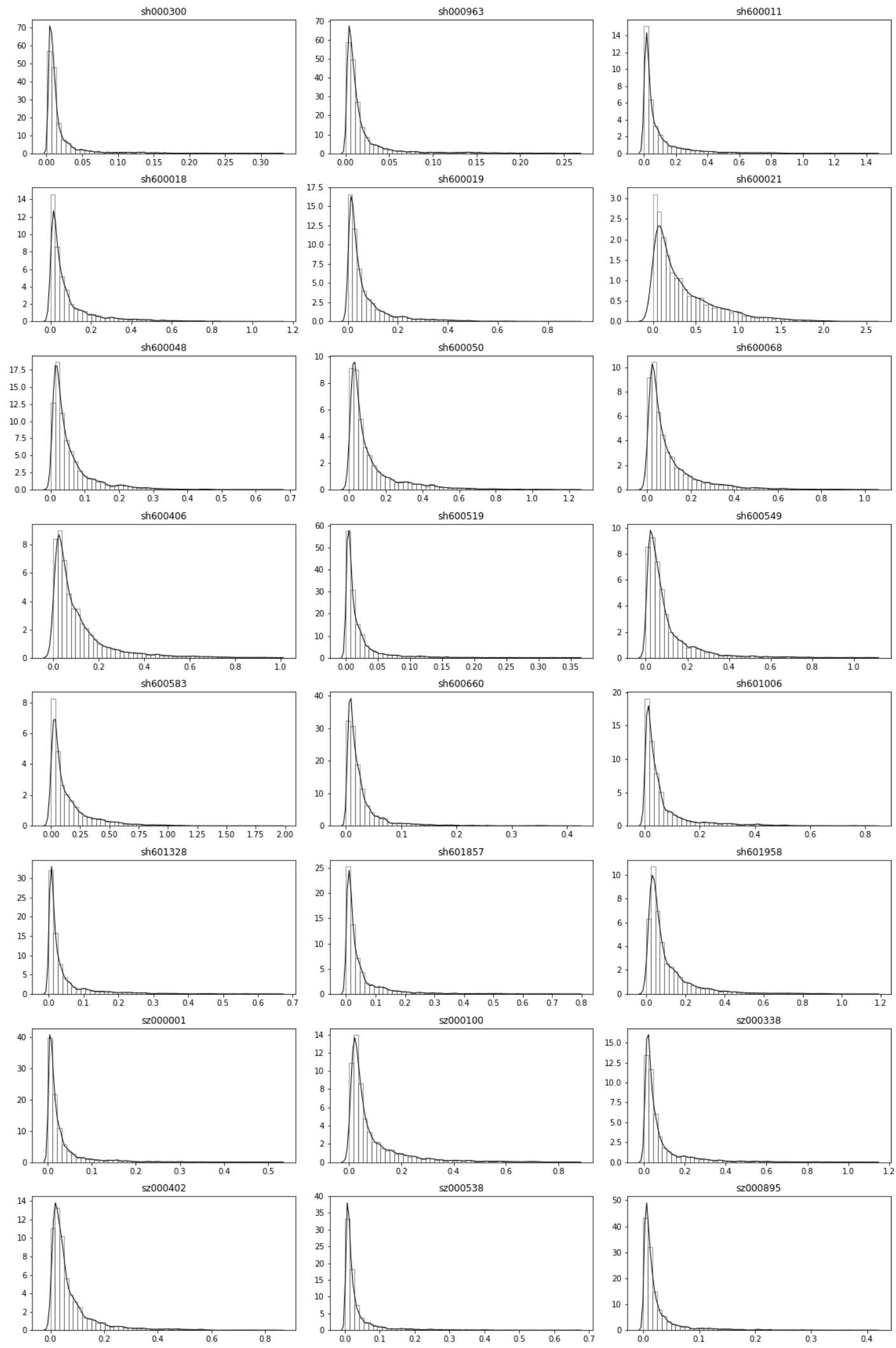

Figure A2. Distribution of $\hat{\lambda}$ of the index and 23 constituent stocks. 